This work is licensed under a Creative Commons Attribution 4.0 International License.

Ovaj rad dostupan je za upotrebu pod licencom Creative Commons Imenovanje 4.0 međunarodna.

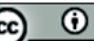

Luka PLANINIĆ

Universität Zadar, Kroatien

Mihovila Pavlinovića, 23000 Zadar

Iplaninic@unizd.hr
UDK 821.112.2.09 Hartmann von Aue-1 DOI: https://doi.org/10.29162/ANAFORA.v8i2.5

Origineller wissenschaftlicher Beitrag

Original Research Article

Erhalten am 20. 5. 2021.

Received: 20 May 2021

Angenommen am 24. 8. 2021

Accepted: 24 August 2021

\title{
KRANKHEIT IN HARTMANNS VON AUE DER ARME HEINRICH
}

\section{Zusammenfassung}

Das Thema „Krankheit“ ist derzeit omnipräsent und aktueller denn je und wird in diesem Beitrag unter verschiedenen Gesichtspunkten am Beispiel von Hartmanns von Aue Der arme Heinrich behandelt. Die bisherigen Forschungen zu dieser Verserzählung legen ihren Fokus vor allem auf die Interpretation der Krankheit im Rahmen einer Sündenstrafe oder einer Prüfung Gottes. Dieser Beitrag erweitert den Blick auf die Krankheitsbilder um die religiöse Thematik und Fragen der Sünde und der Schuld, die das Werk beinhaltet und verdeutlicht ihre Funktion im Kontext der mittelalterlichen Hof- und Rittergesellschaft. Davon ausgehend wird die Rolle der Krankheit in Bezug auf die Darstellung der mittelalterlichen Ständegesellschaft und Standesunterschiede hermeneutisch und literaturwissenschaftlich analysiert und interpretiert. Somit bietet dieser Beitrag eine neue Perspektive in der Forschung der mittelalterlichen Auffassung des Todes, des Glaubens und der Gesellschaft.

Schlüsselwörter: Krankheit, Sünde, Mittelalter, Ritter, Ständegesellschaft 


\section{Einleitung}

Die mittelhochdeutsche Verserzählung Hartmanns von Aue Der arme Heinrich wird oft wegen ihrer Thematik und ihren biblischen Motiven im Kontext der Krankheitsbilder und Religion erforscht und interpretiert. Zwar wird auch in diesem Beitrag auf das Thema der Krankheit eingegangen, jedoch aus einer neuen Perspektive, die bisher nicht in einem genügenden Maß untersucht wurde. Dieser Beitrag widmet sich also der Erforschung der Verbindung zwischen Krankheit und Sünde, die eine zentrale Rolle in dieser Verserzählung spielt. Die bisherigen Forschungen zu diesem Werk legen ihren Fokus meistens auf die Darstellung der Krankheit im Werk in Bezug auf die Rolle Gottes und des Glaubens, da diese Verserzählung deutlich von biblischen Motiven und Themen geprägt ist (vgl. Autoren wie Schirokauer (280), Wapnewski (95) oder Badanes (88)). In diesem Sinne wird häufig auf die Frage eingegangen, ob das Motiv der Krankheit Heinrichs als eine Strafe oder als eine Prüfung Gottes betrachtet werden soll, was aber in diesem Beitrag nicht den Schwerpunkt der Forschung bildet. Hier wird vor allem untersucht, in welcher Verbindung die Krankheit und die Sünde stehen und welche Relevanz diese Verbindung für das Werk hat. Im Vordergrund stehen dabei die Krankheit und die Sünde samt ihren Ursachen und Folgen auf die Entwicklung der Figuren und Handlung sowie die Heilung und die Spiritualität der Figuren in der Verserzählung.

Es wird zuallererst davon ausgegangen, dass die Krankheit in Hartmanns von Aue Der arme Heinrich eine größere Rolle spielt, als es auf den ersten Blick scheint, und nicht nur die körperlichen Störungen und das Leid Heinrichs darstellt, sondern eher eine ganze Gesellschaft, die an falschen Werten und einer zweifelhaften Moral erkrankt ist. Darauf aufbauend soll auf die Thematik der Sünde aufmerksam gemacht werden, denn sie wird auch in diesem Beitrag in Bezug auf die Moral, den Zweifel, die Werte der mittelalterlichen Hof- und Rittergesellschaft und den Aussatz am Beispiel der Figuren und Ereignissen aus der Handlung thematisiert und interpretiert.

\section{Krankheit und Sünde}

Die Erscheinung der Krankheit ${ }^{1}$ wird in der Verserzählung schon am Anfang nach einer kurzen Einleitung beschrieben: „er viel von sînem gebote / ab 
sîner besten werdekeit / in ein smæhlîchez leit: / in ergreif diu miselsuht.“ (von Aue V. 116-119). Kurz davor wird das bevorstehende Leid Heinrichs mit dem biblischen Motiv der Geschichte von Absalom² angekündigt. Dieses Motiv lässt sich an dieser Stelle in der Handlung als ein Vorbote eines künftigen tragischen Schicksals betrachten, denn die Geschichte von Absalom und die vom armen Heinrich ähneln sich nur in einem Aspekt. Das sind die Sünden Heinrichs und Absaloms, die durch die Vergänglichkeit und den trügerischen Schein des irdischen Lebens angedeutet werden: „an im wart erzeiget, / als ouch an Absalône, / daz diu üppige krône / werltlîcher süeze / vellet under die vüeze / ab ir besten werdekeit, / als uns diu schrift hât geseit.“ (von Aue V. 84-90). Es soll nicht unerwähnt bleiben, dass die Sünde im Rahmen dieser Interpretation im engsten Sinne dieses Wortes betrachtet wird, d. h. hier bezieht sich dieser Ausdruck vor allem auf einen Verstoß gegen Menschlichkeit oder gegen Gesellschaft (Eagleton 15). Sowohl Absalom als auch Heinrich weisen Hochmut und Eitelkeit auf, die dann in diesen Erzählungen als Sünden bzw. Übertretungen gegen Gott aufgefasst werden und damit im Kontrast zu den bisherigen Charaktereigenschaften dieser zwei Figuren stehen. In Bezug auf den armen Heinrich dient dieser Kontrast auch der Darstellung von Ursachen der Krankheit. Erst mit der Erklärung der Sünde Heinrichs wird in der Handlung erläutert, dass die Konsequenz seiner Eitelkeit und seines Hochmuts die Lepra bzw. der Aussatz ist: „an hern Heinrîche wart wol schîn: / der in dem hoehsten werde / lebet ûf dirre erde, / derst der versmâhte vor gote. / er viel von sînem gebote / ab sîner besten werdekeit / in ein smæhlîchez leit: / in ergreif diu miselsuht." (von Aue V. 112-119). Es stellt sich aber nun die Frage, wieso die Eitelkeit und der Hochmut dieser Figur auf eine solche Art und Weise bestraft werden. Die Antwort darauf steckt in der Verbindung zwischen der Krankheit und seiner Sünde. Diese Krankheit, die deutlich einen starken Einfluss auf die Figuren in der Verserzählung hat, ist nämlich nur eine Manifestation der Fehler und der Schulden Heinrichs und

chen Wahrnehmung von Krankheiten soll nicht unerwähnt bleiben, dass in dieser Verserzählung nicht das Wort Krankheit im heutigen Sinne des Wortes verwendet wird. Dem Mittelhochdeutschen Handwörterbuch von Matthias Lexer zufolge bezeichnete das mittelhochdeutsche Wort krancheit eher die Schwäche oder die Dürftigkeit einer Person. Stattdessen findet man in Hartmanns von Aue Der arme Heinrich die Bezeichnungen miselsuht (von Aue V. 119), siechtuom (V. 143), swacheit (V. 143) siecheit (V. 166) und suht (V. 232). Das Mittelhochdeutsche Handwörterbuch von Matthias Lexer definiert das mittelhochdeutsche Wort miselsuht als Aussatz oder Lepra in Neuhochdeutsch. Die Wörter siecheit und siechtuom würde man laut diesem Wörterbuch als Krankheit ins Neuhochdeutsche übersetzen und das Wort suht trägt die Bedeutungen Krankheit, Aussatz oder Pest.

${ }^{2}$ Es handelt sich um eine biblische Erzählung von Absalom, dem Sohn des Königs David von Jerusalem, der seinen Vater vom Thron stoßen wollte, aber selbst ein tragisches Ende fand. 
der Gesellschaft, zugleich aber die Erlösung der Figuren, die sich ihrer eigenen Schuld nicht bewusst sind. Die Erkrankung Heinrichs dient nur als Vermittler dieser Schuld, denn durch die Wirkung der Krankheit auf die anderen Figuren in seiner Umgebung kommt seine Schuld erst ans Licht. Das sind aber nicht die einzigen Ursachen für diese Krankheit, sondern nur die offensichtlichsten, die anderen sind erst später in der Handlung zu erkennen und lassen sich mit jeder Figur und ihrer Schuld verbinden.

Ein weiteres Problem, das in der Handlung neben der Eitelkeit Heinrichs behandelt wird, ist seine Vernachlässigung oder sein Verzicht auf Gott und Glauben: „dô nam ich sîn vil kleine war, / der mir daz selbe wunschleben / von sînen gnâden hete gegeben." (von Aue V. 392-394). Schirokauer (280) geht davon aus, dass Lepra als Krankheit in der Verserzählung nicht die Hauptrolle spielt, sondern Heinrich nur den Weg zur Heilung zeigt. In diesem Kontext wird Lepra, als die widerwärtigste der Krankheiten damaliger Zeit neben Pest, laut Schirokauer (280) als ein Mittel zum Zweck verwendet und stellt Heinrichs „Widersetzlichkeit gegen Gott“ bzw. eine „Gotteskrankheit (...) als Strafe für eine extreme Sünde“ dar. Es ist zweifellos, dass diese Krankheit dem armen Heinrich den Weg zur Heilung eröffnet, denn man muss zuerst krank sein, um auch geheilt werden zu können, jedoch steht seine körperliche Lage nicht im Fokus der Verserzählung, sondern seine seelische Krankheit, seine sündige Seele, seine Eitelkeit. Dass Lepra als ein Mittel verwendet wird, mit der Funktion Heinrich zu belehren und ihn zu einer Änderung anzuregen, ist bereits bekannt, sie spielt aber keine Nebenrolle in dem Prozess der Heilung Heinrichs, sondern eben die Hauptrolle. Diese Widersetzlichkeit gegen Gott, die Heinrich zuerst aufweist, ist genau dieselbe Widersetzlichkeit, die er selbst jetzt spüren muss. Sein Körper und seine Seele sind nun so widerwärtig geworden, dass keiner in seiner Nähe bleiben möchte: „dô man die swæren gotes zuht / ersach in sînem lîbe, / manne unde wîbe / wart er dô widerzæme. / nû sehet, wie genæme / er ê der werlte wære, / und wart nû als unmære." (von Aue V. 120-126). Die Lepra ist also hier keine beliebige Krankheit, sondern genau die Darstellung der Sünde und Schuld Heinrichs, die er selbst spüren muss, um sie letztlich auch verstehen und bereuen zu können.

\section{Schuld und Sünde in der Rittergesellschaft}

Die Krankheit spiegelt in dieser Verserzählung nicht nur die seelische Läh338 mung Heinrichs wider, sondern auch die Störungen und die Diskrepanz zwi- 
schen Glauben, Taten und Moral in der Gesellschaft. Das bezieht sich vor allem auf die Hof- und Rittergesellschaft des Hochmittelalters und lässt sich am Beispiel der anderen Figuren aus der Handlung erkennen: durch die Erkrankung Heinrichs offenbaren sich die Heuchelei und die Frömmelei in der Gesellschaft, was am besten am Beispiel der Änderungen in der Umgebung Heinrichs nach seiner Erkrankung zu erkennen ist: „nû sehet, wie genæme / er ê der werlte wære, / und wart nû als unmære. / ze hewe wart sîn grüenez gras, / der ê der werlte venre was, / daz in niemen gerne sach: / als ouch Jôbe geschach, / dem edeln und dem rîchen, / der ouch vil jæmerlîchen / dem miste wart ze teile / iemitten in sinem heile." (von Aue V. 124-132).

Die damalige „Blume der Jugend“ bzw. der zuvor verehrte Ritter Heinrich wird auf einmal aus der höfischen Gesellschaft ausgeschlossen und die Ehre, die er früher genoss, wird durch Scham und Schuld ersetzt. In seiner größten Not verliert Heinrich die Unterstützung und Hilfe seiner Ritter und Freunde. Diese Offenbarung der heuchlerischen Gesellschaft ist an dieser Stelle seiner Krankheit zu verdanken. Die Schuld der Menschen in seiner Umgebung wird durch die Tatsache ersichtlich, dass fast niemand dem armen Heinrich Hilfe leistet, wenn er diese am meisten braucht, somit wendet sich die Gesellschaft gegen Heinrich und verstößt gegen alle ritterlichen Werte ${ }^{3}$, die sie für das Fundament ihrer Gesellschaft hält. Zudem wird dem armen Heinrich dadurch gezeigt, dass sein irdisches Leben und die Vergnügen dieser Welt vergänglich und wertlos sind. Da sein Fokus bisher nur darauf lag, wird er sich an dieser Stelle seiner ursprünglichen Schuld bewusst. Zusätzlich wird diese These in der Handlung durch die parallele Geschichte von $\mathrm{Hiob}^{4}$ gestützt, denn dieser war auch von Gott auf die Probe gestellt, hat aber, im Gegensatz zu Heinrich, alle Herausforderungen von Anfang an mit Geduld und Treue gemeistert. Die Rittergesellschaft wird in Bezug auf diesen Vergleich somit zuerst gepriesen und danach getadelt, denn solange Heinrich dieser Gesellschaft nützlich war, wurde er auch verehrt und als er nicht mehr zur Gesellschaft beitragen konnte, verlor er seinen Platz darin. Zusätzlich wird damit die Doppelmoral der adligen Rittergesellschaft kritisiert und dem armen Heinrich wird durch diese Umkehr

\footnotetext{
${ }^{3}$ Darunter werden in diesem Artikel vor allem die sog. ritterlichen Tugenden wie triuwe, êre, milte, staete, zuht usw. verstanden (vgl. Ehrismann 173).

${ }^{4}$ Die Geschichte von Hiob ist eine biblische Erzählung über das schwere Leiden Hiobs, mit dem Gott die Treue Hiobs auf die Probe stellen wollte. Indem Hiob dieses Leiden ertrug, gelang es ihm zugleich, seine Treue zu beweisen.
} 
der Verhältnisse in seinem Leben verdeutlicht, dass er seinen Fokus auf andere Aspekte seines Lebens und auf andere Menschenwerte legen sollte, wozu ihn seine Krankheit motivieren sollte.

\section{Schuld und Sühne in der Bauernfamilie}

In dieser neuentstandenen Situation gibt es jedoch eine Familie, die ihm $\mathrm{Zu}$ flucht gibt. Genauer gesagt handelt es sich um einen Bauern und seine Familie, deren Herr der Ritter Heinrich ist und die ihm Hilfe leisten, da er ihnen auch immer geholfen und sich ihnen gegenüber gut verhalten hat. Die wichtigste Rolle in der Entwicklung der Handlung und der Problematik von Krankheit und Heilung spielt hier die Tochter des Bauers bzw. das namenlose Bauernmädchen. In diesem Teil der Verserzählung lässt sich ein weiteres Mal der Einfluss der Krankheit in Bezug auf die Verhältnisse einer Gemeinschaft, hier auf die Familienverhältnisse, bemerken. Das heißt die zweifelhafte Moral und die Schuld dieser Familie kommen dank dem leidvollen Zustand Heinrichs ans Licht. Der Bauer und seine Familie gewähren Heinrich nicht nur aus Nächstenliebe Zuflucht, sondern weil ihnen klar ist, dass ohne Heinrich ihr Land und ihr Wohlstand auch verschwinden werden: „waz mac uns mê gewerren / danne an unsern herren, / daz wir den suln verliesen / und mit ime verkiesen / beide guot und êre? / wir gewinnen niemer mêre / deheinen herren alsô guot, / der uns tuo, daz er uns tuot." (von Aue V. 491-498).

Hinsichtlich der früher erwähnten Ursachen der Lepra und der Sünden der Figuren lässt sich diese Tat aber auch als Eigennutz oder sogar als Selbstsucht kennzeichnen. Die Absichten des Bauers und seiner Familie kann man einerseits als Nächstenliebe oder Gnade betrachten, doch ihre edle Hilfe kann andererseits ihre Schuld nicht mildern, denn sie brauchen Heinrich, weil es ihnen gut geht, wenn es auch ihm gut geht. Die Lepra übernimmt in diesem Kontext wieder einmal die Funktion eines Mittels, das die Schuld dieser Familie verdeutlicht. Das Bauermädchen treibt dieses Verhalten jedoch auf die Spitze. Es hat die Absicht, sich für Heinrich zu opfern, damit er dadurch geheilt werden kann. Das Bauernmädchen ist von seiner starken Geistigkeit getrieben, sich selbst, seiner Familie und dem armen Heinrich das Seelenheil und das ewige Leben zu sichern: „ez sol ze Salerne geschehen. / dâ sol der tôt uns loesen / von den hellegeisten boesen. / des tôdes genese wir, / und ich verre baz dan ir." (von Aue V. 852-853). Die Verbindung zwischen Heinrichs Krankheit und 340 der Schuld dieser Familie spiegelt sich an dieser Stelle in der geplanten heroi- 
schen Tat des Bauernmädchens wider, da die Wirkung der Krankheit Heinrichs auf das Bauernmädchen diesen Plan ans Licht bringt. Obwohl auf den ersten Blick seine Absicht sehr edel und lobenswert scheint, handelt das Mädchen möglicherweise nicht nur aus Nächstenliebe. Aus einer anderen Perspektive handelt das Mädchen vermutlich aus dem Eigennutz, d. h. mit dem Ziel vor allem sich selbst das ewige Leben zu sichern und danach auch die eigene Familie und Heinrich zu retten. Darüber hinaus taucht dadurch in diesen Absichten die früher erwähnte Eitelkeit wieder auf, dass das Bauernmädchen selbst die Rolle Gottes übernimmt, indem es die Macht ergreift, über Leben und Tod zu richten. Zudem erkennt man an diesen Handlungsweisen einen Heilsegoismus bzw. einen großen Wunsch, sich selbst das Seelenheil und die „Himmelskrone“ zu gewähren, was auch mehrmals und fast übertrieben von diesem Mädchen hervorgehoben wird: „ez ist mir komen ûf daz zil, / des ich got iemer loben wil, / daz ich den jungen lîp mac geben / umbe daz êwige leben." (von Aue V. 607610).

Den Höhepunkt erreicht dieser Egoismus kurz vor Ende der Handlung, als der arme Heinrich den Tod seiner Braut verhindert. Ihre Wut und Verzweiflung wegen der Himmelskrone, die ihr von Heinrich genommen wurde, kann man als sehr extrem kennzeichnen, denn sie freute sich auf den Tod wie keiner. Dieser Wutanfall bestätigt somit zusätzlich ihre wahren Absichten bzw. ihren Heilsegoismus. Es handelt sich hier weniger um die Gnade Gottes oder die Erlösung Heinrichs, sondern eher um einen Machtrausch einer verzweifelten Jungfrau, die die angestrebte Tat als ihr Schicksal und ihre eigene Rettung empfindet, tatsächlich ist aber ihre Absicht eine Darstellung der Unreife und Naivität, wenn nicht auch des Eigennutzes. Obwohl sie als eine vorbildliche Gläubige betrachtet werden kann und damit auch das Gegenteil Heinrichs hinsichtlich der Geistigkeit und des Glaubens repräsentiert, hat sie sich unbewusst dazu entschlossen, gegen den Willen Gottes zu verstoßen (am Anfang der Handlung wird erklärt, dass Heinrich auf Gottes Geheiß krank geworden ist) und somit eine Sünde zu begehen, was schließlich der Grund für den Untergang ihres Plans war:

dû hâst einen tumben gedanc, / daz dû sunder sînen danc / gerst ze lebenne einen tac, / wider den niemen niht enmac. / du enweist ouch rehte, waz dû tuost, / sît dû benamen ersterben muost, / daz dû diz lasterlîche leben, / daz dir got hât gegeben, / niht vil willeclîchen treist / unde ouch dar zuo niene weist, / ob dich des kindes tôt ernert. / swaz dir got hât beschert, / 
daz lâ allez geschehen. / ich enwil des kindes tôt niht sehen. (von Aue V. 1243-1256)

Dieser heroische Plan des Bauernmädchens scheint vielleicht auf den ersten Blick, die endgültige und lang erwartete Lösung der Problematik von Krankheit zu sein. Trotzdem wird mit den oben genannten Versen darauf hingewiesen, dass eine Sünde nicht mit einer weiteren Sünde gesühnt werden kann. Das heißt, dass sie die Schuld Heinrichs nicht mit eigener Schuld abbüßen soll oder kann. Dieses Problem wird auch in der Verserzählung durch das Gespräch zwischen dem Bauernmädchen und seinen Eltern angekündigt. Die Mutter erklärt dem Mädchen, dass mit seinem Opfer (bzw. mit dem geplanten Selbstmord) gegen das vierte Gebot ${ }^{5}$ des Dekalogs verstoßen wird, was eigentlich ein Verstoß gegen Eltern und Gott wäre und damit würde das Bauernmädchen auch eine Sünde begehen: „wan gedenkestû an sîn gebot? / jâ gebôt er unde bater, / daz man muoter unde vater / minne und êre biete, / und geheizet daz ze miete, / daz der sêle genist werde / und lanclîp ûf der erde. / dû gihst, dû wellest dîn leben / durch unser beider vreude geben: / dû wilt iedoch uns beiden / daz leben vaste leiden." (von Aue V. 640-650).

Die Eltern des Bauernmädchens werden später von jedem Zweifel befreit, indem sie auf die Idee kommen, dass der Heilige Geist selbst durch ihre Tochter spricht, was diese Weisheit, die ein junges Mädchen da aufweist, auch einigermaßen erklären würde. Ob diese Rede wirklich die Worte des Bauernmädchens oder des Heiligen Geistes sind, bleibt in der Verserzählung unklar, doch bezugnehmend auf die Entstehungszeit der Verserzählung und die damalige Ständegesellschaft ist es wenig plausibel, dass ein vermutlich analphabetisches Kind eines unfreien Bauers solche Weisheit und Eloquenz aufweisen würde. Diese Rede des Bauernmädchens lässt sich als eine rhetorische Ironie in der Handlung interpretieren, denn die Heilung Heinrichs erfolgt nicht dank den Begegnungen mit den Ärzten in Salerno und Montpellier, wie man vielleicht erwarten würde, sondern dank den Gedanken und Ideen eines analphabetischen und namenlosen Bauernmädchens. Durch diese Ironie wird die Unangemessenheit der Situation hervorgehoben, denn ein mittelalterlicher Ritter der adligen Hof- und Rittergesellschaft würde nie Zuflucht und Rettung bei einer Bauernfamilie suchen. Diese Ironie dient also der Darstellung einer utopischen Gesellschaft des

${ }^{5}$ Das vierte Gebot lautet: „Ehre deinen Vater und deine Mutter, damit du lange lebst in dem Land, das der Herr, dein Gott, dir gibt.“ 
Mittelalters, die sich aber in der Realität sehr von der damaligen Ständegesellschaft unterscheidet. Die Tatsache, dass der geplante Selbstmord dieses Mädchens später gescheitert ist und dass die Heilung nur durch die Taten Heinrichs erfolgen kann, weist darauf hin, dass der gescheiterte Selbstmord keine Lösung dieser Konfliktsituation sein kann und dass die Heilung nicht durch fremde Hand erfolgen kann, jedoch hat das Mädchen dazu beigetragen, dass Heinrich sich auf den Weg zur Heilung macht.

\section{Eine vorbildliche Gläubige}

Die Rolle des Bauernmädchens soll aus einem weiteren Aspekt näher betrachtet werden und zwar aus seiner Funktion als Vorbild der geistigen Gläubigen und des Gegenteils Heinrichs, die sich am besten in den folgenden Versen beobachten lässt: ,ich vürhte, solde ich werden alt, / daz mich der werlte süeze / zuhte under vüeze, / als sî vil manegen hât gezogen, / den ouch ir süeze hât betrogen; / sô würde ich lîhte gote entsaget.“ (von Aue V. 700-705). Dieser Gedanke des Mädchens stellt den größten Unterschied zu dem armen Heinrich dar, denn es ist von seiner Spiritualität getrieben und lässt sich nicht von dem verführerischen Vergnügen des irdischen Lebens täuschen, während Heinrich in seinem bisherigen Leben den Fokus nur auf die weltlichen Vergnügen richtete. Das ist aber nicht der einzige Unterschied; das Bauernmädchen unterscheidet sich nämlich auch durch seinen Charakter und den Lebensentwurf von Heinrich und stellt somit ein komplettes Gegenteil von ihm dar. Hinsichtlich des Alters, des Geschlechts, des Standes, der Denkweise und der Weltanschauungen sowie des Glaubens stehen diese zwei Figuren in einem Kontrast, der gleichzeitig mindestens zwei Funktionen hat, die dann die Ereignisse aus der Handlung und die Entwicklung der Figuren weiter beeinflussen. Zum einen wird der Konflikt zwischen dem früheren Leben Heinrichs und dem von dem Mädchen angestrebten Leben im Himmel hervorgehoben, womit verdeutlich wird, woran es Heinrich, einem ritterlichen Vorbild, mangelt. Zum anderen ermöglicht dieser Konflikt dem armen Heinrich, die vorbildlichen Werte und Tugenden, die ihm fehlen und deren Abwesenheit zu den Ursachen seiner Krankheit zählt, an einem armen Bauernmädchen zu erkennen, wodurch eine innere Reflexion und Veränderung bei ihm ausgelöst werden. Diese Veränderung wird aber erst mit der Erkrankung Heinrichs vollzogen und zeigt ihm den Weg zur Heilung, was darauf hinweist, dass seine Krankheit auch der Auslöser seiner Heilung ist, die erst dann erfolgt, als er alle Hindernisse auf diesem Weg überwunden bzw. 
als er sich selbst überwunden hat. Gleichzeitig dient die Krankheit Heinrichs auch dem Bauernmädchen, indem ihm gezeigt wird, dass in all dieser Armut das Bauernmädchen seelisch reicher ist als der arme Ritter. Wenn das Mädchen zuvor der Meinung war, dass das Leben Heinrichs wertvoller als sein eigenes war, wird diesem Mädchen jetzt gezeigt, dass es nicht über den Wert einer Seele richten darf, denn dieses obliegt in der Handlung Gott allein, wie hier zu sehen ist: „swaz dir got hât beschert, / daz lâ allez geschehen. / ich enwil des kindes tôt niht sehen." (von Aue V. 1254-1256).

\section{Gegensätze ziehen sich an}

Ähnliche Beispiele kontrastiver Figuren, von denen wenigstens eine als Vorbild oder Erzieher gilt, lassen sich auch in anderen literarischen Werken finden. Hier können beispielsweise die Figuren von Faust und Gretchen aus der berühmten Tragödie Goethes bezüglich der Spiritualität genannt werden, wobei Gretchen mit ihrem Glauben und ihren Weltanschauungen das Gegenteil von Faust darstellt. Darüber hinaus betrachtet man auch in Wolfram von Eschenbachs Parzival die Beziehung zwischen Parzival und seinem Mentor, dem Ritter Gurnemanz, der diesem die ritterlichen Denk- und Handlungsweisen beibringt und ihn dabei auch lehrt, wie man betet und wie man sich als Ritter im Dienst Gottes verhält. Die Bedeutung solcher Beziehungen zeigt sich in den Folgen, die diese Beziehung auf die Entwicklung der Figuren oder Handlung hat. In diesem Fall waren es vor allem die Unreife und die Naivität des Bauernmädchens und der Irrtum samt der Verzweiflung Heinrichs, die sie zusammengefügt haben. Die Folge war ein irreführender Plan, der ihnen beiden Rettung gewähren sollte. Am Ende finden sie beide ihre Rettung, aber nur weil Heinrich im letzten Augenblick aufgeklärt wird und den Tod des Bauernmädchens verhindert. Durch diese letzte Tat der Selbstlosigkeit ist er seine Eitelkeit losgeworden und hat sie durch Demut ersetzt, was er aber dem Bauernmädchen und seinem irreführenden Plan zu verdanken hat. Somit wurde seine körperliche und seelische Krankheit geheilt und das Bauernmädchen hat zugleich eine zweite Chance auf das irdische Leben erhalten, was sie wiederum der Krankheit und der Heilung Heinrichs zu verdanken hat. Zudem sind sie beide ihre Abneigung losgeworden, Heinrich seine gegen Gott und das Bauernmädchen seine gegen das irdische Leben. Folglich haben sie ihre Leben wieder in den Griff bekommen, was ohne die Erkrankung Heinrichs nicht geschehen wäre. Die Krankheit selbst war

nicht genug, sondern sie mussten auch ihre Schuld abbüßen, was sie auch getan 
haben, indem sie Eitelkeit und Eigennutz durch Demut und Selbstlosigkeit ersetzt haben und somit die Probe Gottes bestanden haben: „dô erzeicte der heilic Krist, / wie liep im triuwe und bärmde ist, / und schiet sî dô beide / von allem ir leide / und machete in dâ zestunt / reine unde wol gesunt." (von Aue V. 13651370). Die Relevanz von Krankheit und Sünde in dieser Verserzählung kann auch ganz am Ende der Handlung betrachtet werden, da die Verserzählung mit einem Gebet endet. Damit wird gezeigt, wie diese Figuren das ewige Leben und die Seligkeit im Himmel verdient haben, indem sie die physische und die seelische Krankheit letztendlich bewältigt haben: „nâch süezem lanclîbe / dô besâzen sî gelîche / daz êwige rîche. / alsô müezez uns allen / ze jungest gevallen! / den lôn, den sî dâ nâmen, / des helfe uns got. âmen." (von Aue V. 1514-1520).

Es lässt sich also feststellen, dass die Krankheit in dieser Verserzählung mehrere Funktionen in Bezug auf die Entwicklung der Handlung und der Figuren übernimmt. Eine der Funktionen der Lepra ist die Darstellung einer Prüfung oder Versuchung für die Figuren, worauf die früher erwähnte biblische Erzählung von Hiob sowie die folgenden Verse hinweisen:

Nû hete sich diu guote maget / sô gar verweinet und verklaget, / vil nâhen unz an den tôt. / dô erkande ir triuwe und ir nôt / cordis speculator, / vor dem deheines herzen tor / vürnames niht beslozzen ist. / sît er durch sînen süezen list / an in beiden des geruochte, / daz er sî versuochte / rehte alsô volleclîchen / sam Jôben den rîchen. (von Aue V. 1353-1364)

Der hier erwähnte cordis speculator (der ins Herz schauende) ist in der Handlung nämlich wieder Gott, der den armen Heinrich und die anderen Figuren mit dieser Krankheit auf die Probe stellt. Um diese Prüfung zu bestehen, war es für sie notwendig, einen Rückweg zu Gott zu finden und das Leid mit Geduld zu ertragen und dabei nicht wieder den leichteren und mit Sünden gepflasterten Weg auszuwählen. Auf diese Weise wird es ihnen ermöglicht, eine innere Umkehrung und Heilung der Seele zu erreichen. Um diese Umkehr zu erleben, haben die Figuren anders handeln müssen, als sie es normalerweise tun, damit sich etwas an ihrer Lage ändert. Obwohl diese Umkehrung letztendlich von innen erfolgte, wurde sie zuerst von äußeren Umständen stimuliert, was in diesem Fall nämlich die körperliche Krankheit Heinrichs ist, in der sich dann auch seine seelische Erkrankung und die Erkrankung der anderen Figuren und der Gesellschaft widerspiegelte. Der cordis speculator sieht, wie der Name schon verrät, was in den Herzen dieser Menschen steckt, somit wurde genau das passende 
Mittel gewählt, das ihnen den Weg zur Heilung zeigt. Diese Funktion gehört nochmal der Lepra, die zugleich eine Krankheit und ein Heilmittel ist, wobei das von dem Bauernmädchen angestrebte Opfer nur ein scheinbares Heilmittel darstellt. Der Grund dafür liegt in der Tatsache, dass die Schuld dieser Figuren in ihren Einschränkungen und Handlungsweisen liegt, deswegen müssen sie sich nicht unbedingt mit einer Krankheit, sondern jeweils mit sich selbst und mit den eigenen Fehlverhalten auseinandersetzen. Dementsprechend soll jede dieser Figuren das Heilmittel für die Krankheit bzw. die Lösung für ihr eigenes Leid in sich selbst suchen.

\section{Seuchen und Medizin im Mittelalter}

An dieser Stelle ist zu erwähnen, dass sich die Medizin des Mittelalters, die durch die medizinischen Lehren der Antike in großem Maß geprägt war, besonders an den berühmten medizinischen Hochschulen in Bologna, Salerno und Montpellier, im 12. und 13. Jahrhundert in einem Zustand der Spekulation und Erstarrung befand (Nebel 288). Erst im 14. Jahrhundert beginnt eine anatomische Erforschung des menschlichen Körpers durch das Sezieren des Körpers und anhand von Beschreibungen der Anatomie des Menschen. Daher beruhte diese heute als mangelhaft und lückenhaft betrachtete Medizin oft auf der Religion bzw. auf dem Glauben und dem Aberglauben, weswegen die an Aussatz, Pest oder ähnlichen Krankheiten erkrankten Menschen oft in qualvollen Leiden auf den Tod warteten. Aus Mangel an Wissen und Kenntnissen über Krankheitserreger, Symptome und Heilmittel wendeten sich die Menschen voller Hoffnung auf Rettung an Gott. Aus diesem Grund wurde üblicherweise davon ausgegangen, dass Krankheiten und Pandemien wie beispielsweise die Pest als eine Strafe Gottes zu betrachten sind oder dass solche Krankheiten nur durch Gottes Fügung oder durch ein Wunder geheilt werden können. Eine solche Wahrnehmung der Krankheiten stammt aus der Bibel oder genauer gesagt aus dem Alten Testament, in dem Krankheiten als eine Strafe oder Probe dargestellt werden. (Kolmer 158) Hier spielen Krankheiten wie Pest und Lepra eine wichtige Rolle, denn sie wurden am meisten mit der Rache Gottes verknüpft. Der Grund dafür liegt in der Natur dieser Krankheiten und den Schaden, den sie dem menschlichen Körper zufügen. Tatsächlich handelt es sich um sehr widerwärtige Krankheiten, die den menschlichen Körper von innen nach außen zerfallen lassen, so dass der Körper völlig verdirbt, bis der oder die Kranke unter großen Qualen stirbt (Schirokauer 279). 


\section{Eine schwerkranke Gesellschaft}

Da sich diese Krankheiten, in diesem Fall besonders die Lepra, auf solch grausame Art und Weise manifestieren, dass sie großen Abscheu erregen, wird auch die psychische Gesundheit des Patienten geschädigt. Die Darstellung von Lepra in der Handlung entspricht also in einem großen Maß der damaligen Wahrnehmung von Seuchen und Krankheiten und die Erwähnung von medizinischen Hochschulen in Salerno und Montpellier in der Handlung weist darauf hin, dass sich der Autor in einem gewissen Maß mit den medizinischen Versuchen und Untersuchungen seiner Zeit auskannte. Dennoch entspricht das Motiv des Herzblutes einer heiratsfähigen Jungfrau, das angeblich den armen Heinrich heilen soll, einem wenig plausiblen Heilmittel für diese Krankheit. Trotzdem sind in diesem Motiv die für die mittelalterliche Literatur spezifische Mystik und die übernatürlichen Elemente enthalten. Die biblischen Motive und die deutliche Betonung der Geistigkeit in der Verserzählung sind auch Elemente, die der mittelalterlichen Literatur zuzuordnen sind und kommen in diesem Werk am meisten in den Ursachen der Krankheit und der Schuld der Figuren zum Vorschein.

Die Ursachen dieser Krankheit wurden bisher an mehreren Stellen in diesem Beitrag beleuchtet. Vor allem wird dies mit den Sünden und der Schuld der Figuren in der Handlung verknüpft, die darauf hinweisen, dass jede dieser Figuren eine eigene Schuld abbüßen musste, womit auch die Rittergesellschaft der Heilung einen Schritt näherkommt. Es war nicht nur das Leben Heinrichs und seine Handlungsweisen, die die Notwendigkeit dieser Krankheit bezeugt, sondern auch diese der anderen Figuren, die Sünden wie Eitelkeit, Hochmut, Eigennutz, Selbstsucht, Heuchelei und Frömmelei sühnen mussten. Die Erkrankung Heinrichs löst in dieser Verserzählung eine Kettenreaktion aus, die schließlich allen Figuren in der Handlung das angestrebte Seelenheil gewährt, was zugleich eine Lösung der Problematik dieser Verserzählung bietet und die Versöhnung der in der Handlung behandelten Unterschiede und Diskrepanzen im Leben der Figuren und in der Gesellschaft darstellt. Diese Unterschiede und Probleme in der Gesellschaft, die auf der Doppelmoral und der Heuchelei beruhen, sind vor allem am Beispiel der Standesunterschiede der Figuren zu betrachten. Einerseits handelt es sich um einen Ritter voller ritterlichen Tugenden, der dank der höfischen Erziehung zu einem Rittervorbild wird und somit eine wichtige Rolle in der Gestaltung und Verwaltung dieser Gesellschaft einnimmt. An seinen Handlungsweisen ist aber zu erkennen, dass er Mangel 
an Demut und Opferbereitschaft hat, weswegen er auch ein verzerrtes Bild der Gesellschaft pflegt und somit selbst eine Doppelmoral aufweist. Andererseits geht es um ein Bauernmädchen, das nicht dem Adel, sondern dem Bauernstand bzw. dem niedrigsten Stand dieser Gesellschaft angehört und somit das Gegenteil Heinrichs repräsentiert. Trotzdem wird diesem adligen Ritter das Herzblut eines Bauernmädchens angeboten, damit er geheilt werden kann. Das Blut des Bauernstandes wird also als der Treibstoff des Adels dargestellt, was tatsächlich dem Konzept der Ständegesellschaft entspricht. Die niedrigsten Schichten bzw. die Bauern gewähren dem Adel und dem Klerus ein gemütliches Leben durch die harte Arbeit und das schwere Leben, das sie führen.

In dieser Verserzählung wird dieses Konzept mithilfe einer utopischen Darstellung der Gesellschaft umgestaltet. Selbstverständlich würde diese Geschichte ganz anders aussehen, wenn es die Idee einer idealisierten Hofgesellschaft nicht gäbe. Wenn die Situation umgekehrt wäre bzw. wenn das Bauernmädchen krank wäre, würde sich kaum ein Ritter aus dieser heuchlerischen und hochmütigen Gesellschaft für eine Angehörige des Bauernstandes opfern. Dies wird aber mit einer Einschränkung in der Handlung umgestaltet und zwar, dass sich eine heiratsfähige Jungfrau freiwillig opfern muss, damit Heinrich geheilt werden kann: „ir müeset haben eine maget, / diu vollen manbære / und des willen wære, / daz sî den tôt durch iuch lite." (von Aue V. 224-227). Die Betonung liegt bei dieser Einschränkung also auf der Freiwilligkeit eines Bauernmädchens, sich für einen Ritter zu opfern. Dadurch wird ein solches Opfer als eine heroische Tat dargestellt und die Rittergesellschaft erscheint somit in einem anderen, besseren Licht. Die Wichtigkeit der Rolle der Krankheit in dieser Verserzählung Hartmanns von Aue zeigt sich also vor allem in der Darstellung einer Gesellschaft, die an falschen Werten erkrankt ist und die durch die Taten eines Ritters und eines Bauernmädchens, die beide ihren jeweiligen Stand repräsentieren, geheilt wird. Diese Erforschung der Verbindung von Krankheit und Sünde verdeutlicht die Ungleichheit und die Diskrepanz in der Gesellschaft sowie die Unterdrückung der niedrigsten Schichten, auf denen die damalige Ständegesellschaft beruhte. Durch die Umgestaltung dieser Hof- und Rittergesellschaft wird in der Verserzählung die utopische Idee des Autors von einer idealisierten und balancierten Gesellschaft geschildert. Das ist eine Gesellschaft ohne Standesunterschiede, ohne Herren oder Untertane, eine Gesellschaft in der ein adliger Ritter gerne sein Leben für ein Bauernmädchen opfern würde und nicht umgekehrt, wie es damals zu erwarten war. Mit anderen Worten, es handelt sich 
um eine Gesellschaft, die Gleichheit und Toleranz fördert und damit allen ihren Mitgliedern die gleichen Gelegenheiten und Bedingungen ermöglicht. Eine wichtige Rolle spielt dabei die Religion sowie der Glaube an Gott, da die damalige Hofgesellschaft stark davon geprägt war, was auch aus der Stellungnahme des Autors in dieser Verserzählung zu erschließen ist. Der Grund dafür ist nichts anderes als die Werte, die eine Religion unter ihren Anhängern proklamiert. Werte wie Nächstenliebe, Gleichheit, Frieden, Verzeihung und Hilfsbereitschaft sollten den Anhängern einer Religion ein angenehmes Leben im Gleichgewicht mit sich selbst und mit anderen gewähren. Genau diese Werte, auf denen alle Religionen der Welt in ihrem Kern (ihrer anderen Unterschiede ungeachtet) beruhen, werden in dieser Verserzählung geschildert. Somit sollte diese utopische und von Hartmann von Aue ausgedachte Gesellschaft auf ähnlichen Werten beruhen, damit eine Gesellschaft ohne Unterschiede und Konflikte zwischen verschiedenen Gesellschaftsschichten und ihren Mitgliedern entstehen könnte und nach der sich jeder sehnen würde.

\section{Schlussfolgerung}

Der Ausgangspunkt dieser Forschung war die Hypothese, dass die in Hartmanns von Aue Verserzählung Der arme Heinrich thematisierte Krankheit eine größere Rolle spielt als nur der Gestaltung der Geschichte des armen Heinrichs zu dienen. Diese Idee wurde mit den Fragen der Sünde und der Schuld in Verbindung gebracht und somit wurde in diesem Beitrag die Funktion dieser Verbindung in Bezug auf die Darstellung der Krankheit in der Verserzählung untersucht. Es hat sich beispielsweise erwiesen, dass einer schweren und tödlichen Krankheit wie Lepra, in diesem Werk eine Funktion des Auslösers für Veränderungen zugeschrieben werden kann. Dazu zählen vor allem die Veränderungen der Weltanschauungen und Identitäten der Figuren von Heinrich, dem Bauernmädchen und seiner Familie sowie die Veränderungen in der Ritter- und Ständegesellschaft, deren Vertreter die oben genannten Figuren sind. Beispielsweise hat Heinrich dank dieser Krankheit erkannt, dass er arm an Spiritualität und Demut war. Darüber hinaus wird mit Lepra auf die Krankheit dieser Rittergesellschaft und der höfischen Kultur hingewiesen. Einige Beispiele davon sind das ironisch dargestellte Opfer Heinrichs für das Bauernmädchen sowie die kritische Darstellung von Werten wie Eitelkeit, Hochmut und Heuchelei, auf denen diese Gesellschaft beruht. Neben dieser Kritik der mittelalterlichen Ständegesellschaft, deren Fundament das Blut der niedrigeren Schichten ist, 
schildert von Aue gleichzeitig eine Idee von einer utopischen Gesellschaft ohne Standesunterschiede oder gesellschaftlichen Ungleichheiten. Mithilfe der Ironie und der Umgestaltung der Werte und Handlungsweisen der Figuren wird mit dieser Verserzählung darauf hingewiesen, dass eine ganze Gesellschaft samt ihren Regeln und ihrer Moral geheilt werden sollte.

\section{Literaturverzeichnis}

Badanes, Leslie. “Heinrich's Leprosy: Punishment or Test?” Modern Language Studies, Ausg. 10, Nr. 2, 1980, S. 88-92. JSTOR, www.jstor.org/stable/3194248.

Bibliotheca Augustana, Ulrich Harsch, www.hs-augsburg.de/ harsch/Chronologia/Lspost06/Boethius/boe_con0.html. Abrufdatum 30. 1. 2021.

Bibliotheca Augustana, Ulrich Harsch, www.hs-augsburg.de/ harsch/germanica/Chronologie/12Jh/Hartmann/har_hein.html. Abrufdatum 30. 1. 2021.

Das Wissenschaftliche Bibellexikon im Internet. Alkier, St. et al., www.wibilex.de/. Abrufdatum 31. 1. 2021.

Duden online. Bibliographisches Institut GmbH, www.duden.de/rechtschreibung/Krankheit. Abrufdatum: 30. 1. 2021.

Eagleton, Terry. On Evil. Yale University Press. 2010.

Ehrismann, Gustav. "Die Grundlagen Des Ritterlichen Tugendsystems." Zeitschrift Für Deutsches Altertum Und Deutsche Literatur, Ausg. 56, Nr. 3/4, 1919, S. 137-16. JSTOR, www.jstor.org/stable/20657007.

Kolmer, Lothar. "Heilige Als Magische Heiler." Mediaevistik, Ausg. 6, 1993, S. 153-75. JSTOR, www.jstor.org/stable/42583994.

Mittelhochdeutsches Handwörterbuch von Matthias Lexer. Trier Center for Digital Humanities Kompetenzzentrum, www.woerterbuchnetz.de/?sigle=Lexer\#1. Abrufdatum 29. 1. 2021.

Nebel, Werner. “Zur Geschichte Der Herzdarstellung." Sudhoffs Archiv Für Geschichte Der Medizin Und Der Naturwissenschaften, Ausg. 28, Nr. 4/5, 1935, S. 279-95. JSTOR, www.jstor.org/stable/20773799.

Pschyrembel klinisches Wörterbuch. Walter de Gruyter GmbH, www.pschyrembel.de/Krankheit/K0C8J. Abrufdatum 30. 1. 2021

Schirokauer, Arno. "Die Legende Vom Armen Heinrich." Monatshefte, Ausg. 43, Nr. 6, 1951, S. 279-84. JSTOR, www.jstor.org/stable/30158843.

Von Aue, Hartmann. Der arme Heinrich. Hrsg. von Hermann Paul. Max Niemeyer Verlag. 1958.

Wapnewski, Peter. Hartmann von Aue. J. B. Metzler. 1962. 


\section{ILLNESS IN HARTMANN VON AUE'S \\ DER ARME HEINRICH}

\begin{tabular}{c} 
Abstract \\
\hline Luka PLANINIĆ \\
University of Zadar, Croatia \\
Mihovila Pavlinovića, 23 000 Zadar \\
Iplaninic@unizd.hr \\
\hline
\end{tabular}

The topic of illness is currently omnipresent and more relevant than ever and in this article, it will be approached from various perspectives on the example of Hartmann von Aue's narrative tale Der arme Heinrich. Illness has up to now been researched in this narrative tale either as an interpretation of the divine judgment or as a test of faith. This research offers new insight into depictions of illness in the context of religious subject matter and questions of sin and guilt addressed in this narrative tale, thus clarifying their function within the medieval court society and knighthood. Based on this, a hermeneutic approach and that of literary studies have been chosen in this article in order to analyse and interpret the role of disease regarding the medieval depiction of the estates of the realm and the differences between them. In this manner, the article offers a new perspective concerning the research of medieval perception of death, faith and society.

Keywords: illness, sin, Middle Ages, knights, estates of the realm 


\section{BOLEST U DJELU DER ARME HEINRICH \\ HARTMANNA VON AUEA}

\begin{tabular}{c} 
Sažetak \\
\hline Luka PLANINIĆ \\
Sveučilište u Zadru, Hrvatska \\
Mihovila Pavlinovića, 23 000 Zadar \\
Iplaninic@unizd.hr \\
\hline
\end{tabular}

Bolest kao tema sveprisutna je i aktualnija nego ikada prije, stoga se u radu obrađuje iz različitih gledišta na primjeru djela Der arme Heinrich spisatelja Hartmanna von Auea. Dosadašnja istraživanja bolesti u navedenom djelu usmjerena su ponajprije na tumačenje bolesti kao kazne za grijehe ili Božje kušnje, no ovaj rad nudi nov pogled na prikaze bolesti u kontekstu religiozne tematike te pitanja grijeha i krivnje kojima se djelo bavi i tako razjašnjava njihovu funkciju u kontekstu srednjovjekovnoga dvorskog i viteškoga društva. Na temelju toga u članku se sa stajališta hermeneutike i znanosti o književnosti uloga bolesti proučava i tumači s obzirom na prikazano srednjovjekovno staleško društvo i staleške razlike te se time daje nova perspektiva u istraživanju srednjovjekovnog poimanja smrti, vjere i društva.

Ključne riječi: bolest, grijeh, srednji vijek, vitezovi, staleško društvo 